ICN-UNAM-97-10

1st July, 1997

\title{
Self-Adaptation in Evolving Systems
}

\author{
C.R. Stephens ${ }^{1}$, I. García Olmedo ${ }^{2}$, J. Mora Vargas ${ }^{3}$ \\ and H. Waelbroeck ${ }^{1}$ \\ 1 Instituto de Ciencias Nucleares, UNAM \\ Circuito Exterior, A. Postal 70-543, \\ México D.F. 04510 \\ ${ }^{2}$ Lab. de Visualización, DGSCA, UNAM \\ Circuito Exterior, C.U. \\ México D.F. 04510 \\ ${ }^{3}$ Facultad de Ingeniería, UNAM \\ Circuito Exterior, C.U. \\ México D.F. 04510
}

\begin{abstract}
A theoretical and experimental analysis is made of the effects of self-adaptation in a simple evolving system. Specifically, we consider the effects of coding the mutation and crossover probabilities of a genetic algorithm evolving in certain model fitness landscapes. The resultant genotype-phenotype mapping is degenerate, there being no direct selective advantage for one probability versus another. We show that the action of mutation and crossover breaks this degeneracy leading to an induced symmetry breaking among the genotypic synonyms. We demonstrate that this induced symmetry breaking allows the system to self-adapt in a time dependent environment.
\end{abstract}




\section{Introduction}

One of the most striking features of complex systems, especially in the biological realm, is the ability to adapt. Loosely speaking, this means "optimization" in a time and/or position dependent "environment". The Darwinian paradigm of natural selection offers an intuitive framework within which one may try to understand such adaptive behaviour. Recently, another alternative, or rather complementary, paradigm has been suggested [1] that takes as its principal theme - "spontaneous ordering". In this case advantageous or disadvantageous characteristics may appear via a "spontaneous" symmetry breaking. The principal theme of this paper is to show that adaptation can also occur due to an induced symmetry breaking - induced by the action of the genetic operators other than selection. We will demonstrate the above in the context of the evolution of a genetic algorithm (GA) seen as a simple, artificial model of an evolving, adaptive complex system.

Evolutionary algorithms, and in particular GAs, have played an increasingly important role in a wide variety of problems (see for example [2] for a recent review). Two crucial ingredients of such algorithms are: i) the existence of a population of bit strings/chromosomes, each one of which codifies directly or indirectly a possible solution to a problem; and ii) a set of genetic operators which act on the strings. In the case of GAs the most popular and most studied operators are selection, mutation and crossover, though of course in principle the list is infinite. Many other evolutionary algorithms, such as evolutionary programming [3], rely solely on selection and mutation. It has been asserted that GAs are better at function optimization than evolutionary algorithms that rely solely on mutation and that this advantage is due to crossover 《4.

A lot of effort has been spent trying to understand the differing roles of selection, mutation and crossover in the context of different fitness landscapes, and in particular on discovering what constitute optimal values for the exogenous parameters, e.g. mutation probability, crossover probability etc. [5]. The chief motivation behind this work is to find parameter values which maximise performance over a wide class of test functions. However, it has been shown [6] that effectively the most efficient parameter settings must depend on the specific function.

Intuitively it is clear that time dependent parameters should be utilized as will be illustrated clearly in this paper. In this regard it is possible to specify a priori a schedule for the parameter changes, as is done in simulated annealing with a cooling schedule. A more attractive possibility, however, is to code the parameters within the chromosomes themselves and allow the system to "self-optimize" via a genetic search through various possible parameter values. We will refer to the continual adjustment of these parameters according to changes in the landscape as "self-adaptation". Such selfadaptation first appeared in the context of evolution strategies [7] and has been studied by Bäck and collaborators $[8]$ in both the context of evolution strategies and GAs. One of the prime points of interest was the derivation of optimal mutation rates on a string by string basis in the case of certain simple landscapes. Crossover has been considered in [9] where the actual crossing points were codified in the strings. Codification of the 
crossover probability itself was not considered.

In this paper we will consider the effects of parameter codification of a GA in a set of model fitness landscapes. Our principal aim is not to show that parameter codification can lead to much better function optimization capabilities in GAs, though this may well be the case, but rather to study how self-adaptation occurs in a simple complex system. This self-adaptation will be shown to come about not via the mechanism of Darwinian natural selection but via an induced symmetry breaking.

\section{Optimal Mutation and Crossover Rates}

In this section we will make some observations about optimal parameter settings based on an analysis of the evolution equation, valid in the large population limit, for GAs derived in [10] that takes the following form

$$
P\left(C_{i}, t+1\right)=\mathcal{P}\left(C_{i}\right) P_{c}\left(C_{i}, t\right)+\sum_{C_{j} \neq C_{i}} \mathcal{P}\left(C_{j} \rightarrow C_{i}\right) P_{c}\left(C_{j}, t\right)
$$

where

$$
\begin{aligned}
P_{c}\left(C_{i}, t\right)= & P^{\prime}\left(C_{i}, t\right)-\frac{p_{c}}{N-1} \sum_{C_{j} \neq C_{i}} \sum_{k=1}^{N-1} \mathcal{C}_{C_{i} C_{j}}^{(1)}(k) P^{\prime}\left(C_{i}, t\right) P^{\prime}\left(C_{j}, t\right) \\
& +\frac{p_{c}}{N-1} \sum_{C_{j} \neq C_{i}} \sum_{C_{l} \neq C_{i}} \sum_{k=1}^{N-1} \mathcal{C}_{C_{j} C_{l}}^{(2)}(k) P^{\prime}\left(C_{j}, t\right) P^{\prime}\left(C_{l}, t\right)
\end{aligned}
$$

$p_{c}$ is the probability to implement crossover in the first place, $k$ is the crossover point, and the coefficients $\mathcal{C}_{C_{i} C_{j}}^{(1)}(k)$ and $\mathcal{C}_{C_{j} C_{l}}^{(2)}(k)$ are given by

$$
\mathcal{C}_{C_{i} C_{j}}^{(1)}(k)=\theta\left(d_{L}^{H}(i, j)\right) \theta\left(d_{R}^{H}(i, j)\right)
$$

and

$$
\mathcal{C}_{C_{j} C_{l}}^{(2)}(k)=\frac{1}{2}\left[\delta\left(d_{L}^{H}(i, j)\right) \delta\left(d_{R}^{H}(i, l)\right)+\delta\left(d_{R}^{H}(i, j)\right) \delta\left(d_{L}^{H}(i, l)\right)\right]
$$

where $d_{R}^{H}(i, j)$ is the Hamming distance between the right halves of the strings $C_{i}$ and $C_{j}$, "right" being defined relative to the crossover point. The other quantities in (3) and (国) are defined analogously. $\theta(x)=1$ for $x>0$ and is 0 for $x<0$, whilst $\delta(x)=0 \forall x \neq 0$ and $\delta(0)=1 . P^{\prime}\left(C_{i}, t\right)=\left(f\left(C_{i}, t\right) / \bar{f}(t)\right) P\left(C_{i}, t\right)$ with $f\left(C_{i}, t\right)$ being the fitness of string $C_{i}$ at time $t$, and $\bar{f}(t)=\sum_{i} f\left(C_{i}, t\right) P\left(C_{i}, t\right)$ is the average string fitness. The effective mutation coefficients are $\mathcal{P}\left(C_{i}\right)=\prod_{k=1}^{N}(1-p(k))$, which is the probability that string $i$ remains unmutated, $p(k)$ being the probability of mutation of bit $k$, which we assume to be a constant, though the equations are essentially unchanged if we also include a dependence on time. $\mathcal{P}\left({ }_{C_{j} \rightarrow C_{i}}\right)$ is the probability that string $j$ is mutated into string $i$,

$$
\mathcal{P}\left(C_{j} \rightarrow C_{i}\right)=\prod_{k \in\left\{C_{j}-C_{i}\right\}} p(k) \prod_{k \in\left\{C_{j}-C_{i}\right\}_{c}}(1-p(k))
$$


where $\left\{C_{j}-C_{i}\right\}$ is the set of bits that differ between $C_{j}$ and $C_{i}$ and $\left\{C_{j}-C_{i}\right\}_{c}$, the complement of this set, is the set of bits that are the same. In the limit where the mutation rate $p$ is uniform, $\mathcal{P}\left({ }_{C_{i}}\right)=(1-p)^{N}$ and $\mathcal{P}\left({ }_{C_{j} \rightarrow C_{i}}\right)=p^{d^{H}(i, j)}(1-p)^{N-d^{H}(i, j)}$, where $d^{H}(i, j)$ is the Hamming distance between the strings $C_{i}$ and $C_{j} \cdot \mathcal{C}_{C_{i} C_{j}}^{(1)}(k)$ is the probability that given that $C_{i}$ was one of the parents it is destroyed by the crossover process. $\mathcal{C}_{C_{j} C_{l}}^{(2)}(k)$ is the probability that given that neither parent was $C_{i}$ it is created by the crossover process.

This evolution equation takes into account exactly, given the approximation of a large population, the effects of destruction and reconstruction of strings. By realizing that string reconstruction via crossover takes place via the concatenation of two strings, one of which reconstructs the left part of the desired string, $C_{i}^{L}$, and the other the right part, $C_{i}^{R}$, one can write the equation in a much more elegant form by using

$$
P_{c}\left(C_{i}, t\right)=P^{\prime}\left(C_{i}, t\right)-\frac{p_{c}}{N-1} \sum_{k=1}^{N-1}\left(P^{\prime}\left(C_{i}, t\right)-P^{\prime}\left(C_{i}^{L}, t\right) P^{\prime}\left(C_{i}^{R}, t\right)\right)
$$

with

$$
P^{\prime}\left(C_{i}^{L}, t\right)=\sum_{C_{j} \supset C_{i}^{L}} P^{\prime}\left(C_{j}, t\right)
$$

and similarly for $P^{\prime}\left(C_{i}^{R}, t\right)$.

We may now use the above equation to investigate the evolution of any function. One of particular interest is the increment in average population fitness per generation

$$
\delta \bar{f}=\bar{f}(t+1)-\bar{f}(t)
$$

which using the evolution equation (1) we can write as

$$
\delta \bar{f}=\sum_{C_{i}} f\left(C_{i}, t\right) \mathcal{P}\left(C_{i}\right) P_{c}\left(C_{i}, t\right)+\sum_{C_{i}} \sum_{C_{j} \neq C_{i}} f\left(C_{i}, t\right) \mathcal{P}\left(C_{j} \rightarrow C_{i}\right) P_{c}\left(C_{j}, t\right)-\sum_{C_{i}} f\left(C_{i}, t\right) P\left(C_{i}, t\right)
$$

Note that this equation is highly non-linear in the mutation rate $p$, but linear in the crossover probability $p_{c}$.

The values of $p$ and $p_{c}$ play a very important role in determining the success of a GA. The specific values required in a particular problem however will depend on what we mean by success. There are several different ways in which one may evaluate the success of a particular algorithm. One would be to maximise the average population fitness, $\bar{f}$, generation by generation. Another would be the success probability [8]

$$
S^{+}\left(\left\{p_{i}\right\}\right)=\mathcal{P}(f(p(g))>f(g))
$$

where $\left\{p_{i}\right\}$ represents the genetic operator probabilities and $p(g)$ is the image of the genotype $g$ under the action of all genetic operators. Of course, one can restrict the definition of the success probability to be associated with a particular genetic operator.

Here we will concentrate on the average fitness improvement. As $\delta \bar{f}$ depends only linearly on $p_{c}$, the value of $p_{c}$ that optimizes fitness growth over one time step, if we neglect finite size effects, will be either 0 or 1 . This is somewhat deceptive however in 
that the integrated fitness gain, $\bar{f}(t)-\bar{f}(0)$, will be highly non-linear in $p_{c}$ except when $t=1$. For a given time step however, from (6) one can see that whether or not $p_{c}$ should be minimized or maximized depends on whether parts of highly fit strings are positively or negatively correlated in the selected parent population. If the correlation is negative, i.e. $P^{\prime}\left(C_{i}, t\right)<P^{\prime}\left(C_{i}^{L}, t\right) P^{\prime}\left(C_{i}^{R}, t\right)$, then the reconstruction of highly fit strings dominates over destruction and $p_{c}$ should be maximized. To illustrate this we restrict attention for the moment to the case $p=0$. One finds

$$
\delta \bar{f}=\Delta \bar{f}-\frac{p_{c}}{N-1} \sum_{k=1}^{N-1} \sum_{C_{i}} f\left(C_{i}, t\right)\left(P^{\prime}\left(C_{i}, t\right)-P^{\prime}\left(C_{i}^{L}, t\right) P^{\prime}\left(C_{i}^{R}, t\right)\right)
$$

where $\Delta \bar{f}(t)=\left(\bar{f}^{2}-(\bar{f})^{2}\right) / \bar{f}$ is the variance in the population fitness. As is well known $\Delta \bar{f}(t) \geq 0$, i.e. fitness in the absence of other genetic operators is a Lyapunov function. As stated, whether or not crossover helps or hinders fitness growth depends on whether reconstruction or destruction of highly fit strings is dominant.

We can get some idea of when this occurs by restricting attention to the very simple case of a population of two bit strings (one can also think of this in terms of two schematas). One finds

$$
\delta \bar{f}=\Delta \bar{f}-\frac{p_{c}}{\bar{f}^{2}}\left(f_{00}+f_{11}-f_{10}-f_{01}\right)\left(f_{10} P_{10} f_{01} P_{01}-f_{00} P_{00} f_{11} P_{11}\right)
$$

For crossover to be a net positive force we require either

$$
f_{00}+f_{11}>f_{10}+f_{01} \quad \text { and } \quad f_{10} P_{10} f_{01} P_{01}>f_{00} P_{00} f_{11} P_{11}
$$

or

$$
f_{00}+f_{11}<f_{10}+f_{01} \quad \text { and } \quad f_{10} P_{10} f_{01} P_{01}<f_{00} P_{00} f_{11} P_{11}
$$

For a linear fitness landscape, as $f_{00}+f_{11}=f_{10}+f_{01}$, crossover in this 2-bit problem is neutral, although we once again emphasize that this is at the level of one time step. An interesting corollary of this is that even in the presence of crossover $\bar{f}$ is a Lyapunov function. However, for a deceptive landscape of type I or type II [11] one finds that the effect of crossover is destructive. In this case having low values of $p_{c}$ would be beneficial. Of course if finite size effects are important having $p_{c}$ too low may inhibit genetic diversity.

We can try to generalize this lesson beyond the case of two-bit strings. In (11) one may see that in the sum over strings, if the landscape is deceptive in the sense that $P^{\prime}\left(C_{i}^{L}, t\right) P^{\prime}\left(C_{i}^{R}, t\right)<P^{\prime}\left(C_{i}, t\right)$, then crossover will inhibit fitness growth; whilst if $P^{\prime}\left(C_{i}^{L}, t\right) P^{\prime}\left(C_{i}^{R}, t\right)>P^{\prime}\left(C_{i}, t\right)$ it will enhance fitness growth. In the former case a low $p_{c}$ would be beneficial whilst in the latter a high value. Generally speaking, the more deceptive the landscape the lower the optimum value of $p_{c}$. In this sense GA hard problems may be made GA easier by a reduction in the crossover probability. Note also that we may have deception with respect to one crossover point but not with respect to another; this would imply that a crossover probability, $p_{c}(k)$, that depends on the 
crossover point might well be useful. The point of the above is to show that what constitutes an optimal value for crossover is very much landscape dependent, moreover it may very well be time dependent in that at one time the population may be much more deceptive than at another time.

We now turn our attention to mutations. Once again for simplicity we will turn off the other genetic operator and consider mutations in isolation. We will also assume that $p$ is constant. In this case

$$
\delta \bar{f}=\sum_{C_{i}} \sum_{C_{j}} f\left(C_{i}, t\right) P^{\prime}\left(C_{j}, t\right) p^{d^{H}(i, j)}(1-p)^{N-d^{H}(i, j)}-\sum_{C_{i}} f\left(C_{i}, t\right) P\left(C_{i}, t\right)
$$

thus

$$
\frac{d \delta \bar{f}}{d p}=\sum_{C_{j}} \sum_{C_{j}} f\left(C_{i}, t\right) P^{\prime}\left(C_{j}, t\right) p^{d^{H}(i, j)-1}(1-p)^{N-d^{H}(i, j)-1}\left(d^{H}(i, j)-p N\right)
$$

We now wish to find the extrema. Doing the explicit sums over $C_{i}$ is of course very difficult. One solution is to choose $p^{*}=d^{H}(i, j) / N$, however, this requires making $p$ string specific. One can get an estimate of an optimum $p$ by solving $d \delta \bar{f} / d p=0$ in a mean field approximation where we replace $d^{H}(i, j)$ by $\left\langle d^{H}(i, j)>\right.$, its expectation value over the population. This results in $p^{*}=<d^{H}(i, j)>/ N$. For a random population, where $<d^{H}(i, j)>\sim N / 2, p^{*} \sim 1 / 2$ which accords with intuition. Near the ordered limit where $<d^{H}(i, j)>\sim 0, p^{*} \sim 0$ which once again accords with intuition. Clearly however $p^{*}$ will be time dependent. Note that whether or not zero mutation rate in the ordered limit is optimal or not will depend on whether the population has ordered about the global optimum or a local optimum. In the case of the latter $p=0$ will hinder rather than help.

The point of the above is that different values of the GA parameters can lead to very different performances. Trying to determine optimal parameter values empirically is extremely difficult therefore one is naturally led to the idea of allowing the GA to optimize itself via a codification of the parameters.

\section{Parameter Codification and Symmetry Breaking}

Parameter codification can be done in various ways. Here we choose the simple route of extending the size of the chromosome such that a part of the chromosome now carries information on the mutation and/or crossover probabilities. We assume that the fitness of a chromosome is not affected directly by the values of the parameters. This means that the genotype-phenotype mapping is now non-injective.

Consider the different classes of maps that may be defined: first, $f_{G}: G \longrightarrow R^{+}$ where $G$ denotes the space of genotypes and $f_{G}$ is the fitness function that assigns a number to a given genotype; second, $f_{Q}: \longrightarrow R^{+}$, where $Q$ is the space of phenotypes. It should be emphasized that these mappings may also be explicitly time dependent. In fact this will normally be the case when the "environment" is time dependent. The maps may be injective or surjective. If they are non-injective then there exist "synonomous" 
genotypes or phenotypes, i.e. there is "redundancy" in the mapping. If we assume there exists a map $\phi: G \longrightarrow Q$ between genotype and phenotype then we have $f_{G}=f_{Q} \circ \phi$, i.e. the composite map induces a fitness function on $G$. The map $\phi$ we may fruitfully think of as being an "interpreter", in that the map translates the genotypic information into something we call the phenotype, where generically the fitness function will have a more intuitive interpretation.

One of the principle reasons for using an interpreter in GAs, is that, given a set of genetic operators, the original genotypic coding may not be the most efficient. This is the case for a binary coding in the standard scenario where selection, mutation and simple crossover are the preferred operators. It has been found that a Gray coding [12] that maps Euclidean neighbourhoods into Hamming neighbourhoods is more effective [13.

In the problem at hand, where we are considering codification of parameters, $G$ is simply the set of chromosomes, including the parts that code for the parameter values. $Q$ is now the space of truncated chromosomes where the genes that code for the different parameter values have been removed. The interpreter here is clearly non-injective. If we use an $n_{c}$-bit binary codification of the parameters then the interpreter, and hence the genotypic fitness landscape, will have a $2^{n_{c}}$-fold degeneracy, i.e. for every phenotype there will be $2^{n_{c}}$ corresponding genotypes.

This genotypic symmetry would be broken spontaneously in a finite breeding pool, by the theory of branching processes, this observation being the backbone of the Neutral Theory of molecular evolution [14]. However, there will also be an induced symmetry breaking from the violation of the synonym symmetry by the genetic operators themselves.

If one considers the growth of a particular bit over time selection forces will take into account not only the selective advantage of this bit but also its ability to produce well-adapted offspring, which can themselves produce well-adapted offspring, etc. Since mutation and crossover act differently on synonymous bits, the synonyms will differ in their descendence, both in the passive sense of surviving mutations, and in the active sense of generating new genetic solutions. This implies that the time-averaged effective fitness function, defined as the growth rate of a bit over many generations, does not respect the synonym symmetry. Thus, the effective fitness function provides a selective pressure which enhances the production of potentially successful mutants by selecting, among the synonyms, those that have a higher probability to generate well-adapted offspring.

We will now discuss in the context of a very simple model the phenomenon of induced symmetry breaking. The model we consider consists of three possible genotypes, $A, B$ and $C$, where each genotype can mutate to the two adjacent genotypes. $A$ and $C$ are synonyms in that they encode the same phenotype $a$, i.e. $\phi(A)=\phi(C)=a$, whilst $B$ encodes the phenotype $b$. In a random population, $p(A)=p(B)=p(C)=\frac{1}{3}$. If there is probability $\mu_{1} / 2$ for $A$ to mutate to $B$ or $C$ and probability $\mu_{2} / 2$ for all other possible mutations then the evolution equation that describes this system in the large population 
limit is

$$
P_{i}(t+1)=\left(1-\mu_{i}\right) P_{i}^{\prime}(t)+\left(\mu_{i-1} P_{i-1}^{\prime}(t)+\mu_{i+1} P_{i+1}^{\prime}(t)\right)
$$

$P_{i}(t)$ being the population fraction of genotype $i . \mu_{i}$ is the probability of mutation from genotype $i$ to any other genotype, whilst $\mu_{i-1}$ and $\mu_{i+1}$ are the mutation rates from genotypes $(i-1)$ and $(i+1)$ to genotype $i$ respectively. If we assume a simple fitness landscape, $f_{a}=2, f_{b}=1$, then for $\mu_{i}=0$ the steady state population is $P(A)=$ $P(C)=1 / 2$ and $P(B)=0$. Thus we see the synonym symmetry is unbroken. However, for $\mu_{i}>0$, the genotype distribution at $t=1$ starting from a random distribution at $t=0$ is: $P(A)=\left(4-4 \mu_{1}+3 \mu_{2}\right) / 10, P(B)=\left(1+\mu_{1}\right) / 5, P(C)=\left(4+2 \mu_{1}-3 \mu_{2}\right) / 10$. Thus we see that there is an induced breaking of the synonym symmetry due to the effects of mutation. The full effect can be dramatically seen in Figure 1 where we have $\mu_{1}=0.1, \mu_{2}=0.01$. Clearly, the less mutable string $C$ is strongly favoured over the other synonym $A$.

The effective fitness [10] defined via

$$
P_{i}(t+1)=\frac{f_{e f f_{i}}(t)}{\bar{f}(t)} P_{i}(t)
$$

is from the evolution equation (11) given by

$$
f_{\text {eff }}\left(C_{i}, t\right)=\frac{\bar{f}}{P\left(C_{i}, t\right)}\left(\mathcal{P}\left(C_{i}\right) P_{c}\left(C_{i}, t\right)+\sum_{C_{j} \neq C_{i}} \mathcal{P}\left(C_{j} \rightarrow C_{i}\right) P_{c}\left(C_{j}, t\right)\right)
$$

Explicitly in this model

$$
f_{\text {eff }}(t)=f_{i}+\frac{1}{P_{i}(t)}\left(\mu_{i-1} f_{i-1} P_{i-1}(t)+\mu_{i+1} f_{i+1} P_{i+1}(t)-\mu_{i} f_{i} P_{i}(t)\right)
$$

At $t=0, f_{\text {eff }}(0)=\left(4-4 \mu_{1}+3 \mu_{2}\right) / 2, f_{\text {eff }}(0)=\left(2+2 \mu_{1}-\mu_{2}\right) / 2$ and $f_{\text {eff }}(0)=$ $\left(4+4 \mu_{1}-3 \mu_{2}\right) / 2$. For the case $\mu_{1}=0.1, \mu_{2}=0.01 ; f_{\text {eff }}(0)=1.815, f_{\text {eff }}(0)=1.095$ and $f_{\text {eff }}(0)=2.195$. Thus as mentioned above we see that the effective fitness function provides a selective pressure by selecting among the synonyms those that have a higher probability to produce fit descendents.

\section{Methodology}

In this section we will discuss the model problems we chose to illustrate the phenomenon of parameter adaptation. We will show that parameter codification yields significantly different results to those of a fixed parameter value GA in the following cases: a generic non-deceptive multimodal function; a deceptive function; a time dependent function; a travelling salesman problem of 33 cities and finally a time dependent travelling salesman problem. Throughout we used roulette wheel selection except at one point, which will be explicitly mentioned, where we used tournament selection of size 5 . 
The simple multimodal function is seen in Figure 2. Rather than worry about about how to design a real valued, continuous, double peaked function and then approximate it by binary numbers we simply assigned a fitness value to every integer between 0 and 63 . This function is not deceptive in that crossover between optimal or near optimal strings does not produce very unfit strings, i.e. crossover of strings near 10 and near 40 does not tend to produce strings between 20 and 30, or between 50 and 63. The GA we then used was associated with a fixed population of 500 individuals. The basic chromosome was a 6-bit binary string representing the integers between 0 and 63 . In coding the mutation and/or crossover probabilities we ranged between 3 and 16-bit additions to the basic chromosome depending on whether one or both parameters were coded and whether or not they were coded using 3-bit or 8-bit binary representations, the latter obviously giving a finer representation. The performances of various GAs with and without parameter codification were then tested. In comparing with the performance of a standard GA we chose mutation and crossover probabilities of 0.01 and 0.8 respectively, these being the generally recommended values in the literature [5].

The second landscape we chose to investigate is shown in Figure 3. The landscape is deceptive in the sense that crossover between fit strings associated with the optima $000000=0$ and $111111=63$ produces very unfit strings. The same tests were carried out as with the previous landscape.

The third landscape chosen included time dependence. The initial landscape is that shown in Figure 2, which has as global optimum 10 and 11 . However, after $60 \%$ of the population reach the global optimum the landscape is suddenly changed to that of Figure 4 wherein the original global optimum is now only a local optimum and a new, very narrow global optimum appears at 62 . We call this a "jumper" landscape. In tests with the jumper landscape we used tournament selection of size 5 and also imposed a lower bound of 0.005 for mutation.

The above landscapes were all chosen to illustrate clearly various advantages of parameter codification in a context where the optima are all explicitly known. As an example of a system where the optima are not a priori known and where the size of the state space is very large we considered a 33-city travelling salesman problem (TSP). The TSP is a prototypical NP-complete problem which is easy to state but difficult to solve. Here our aim was to compare the performance of a GA with fixed probabilities for the genetic operators with the same GA but with coded probabilities, not to find the best codification and set of genetic operators for applying GAs to the TSP. It is known that GAs are quite competitive with other optimization techniques [15] when coded appropriately.

With that in mind we chose the simplest minded codification via a path representation wherein the cities are listed in the order they are visited. The genetic operators used were: "mutation" (permutation of two randomly selected cities ) and "crossover" (inversion of the cities between two randomly selected points on the chromosome). For example: for a 6 city problem mutation at 0 and 3 of the possible route 134520 leads to 534120. Inversion between points 2 and 5 of the same route leads to 132540 . We did not bother to avoid cyclic permutations of a given route as once again our principal aim 
is to compare the performance of a given GA with and without parameter codification. The operator probabilities were coded using 8 bits for each one resulting in a 49-bit chromosome. Although two-city interchange and inversion were used on the 33 bits that code for the route standard mutation and crossover were used on the remaining 16 bits. For mutations we set a lower bound of 0.001 rather than zero.

The fitness function for the $n$-city TSP, as is well known, is just the total distance of a route

$$
R=\sum_{i=0}^{n-1}\left(d\left(c_{i}, c_{i+1}\right)\right)+d\left(c_{0}, c_{n-1}\right)
$$

with $d(x, y)$ the distance between cities $x$ and $y$, subject to the restrictions that all cities must be visited and no city can be visited more than once.

Finally, we considered the above problem but in a time dependent context where starting with a TSP of 23 cities at generation 3000 ten more cities were added so that a new optimum route completely different to the first had to be found. To compare the different performances of the GAs in the TSP we used the following function

$$
\mathcal{F}=\sum_{i=j}^{j+100} \frac{R_{\min }(i)+R_{a v}(i)}{R_{\min }(i)-R_{a v}(i)}
$$

where the sum is over an interval of 100 generations and $j$ represents the discrete time at which we are evaluating $\mathcal{F}$. Thus over a period of 6000 generations we will have a set of 60 points to evaluate $\mathcal{F}$. $R_{\text {min }}(i)$ is the shortest route length found at generation $i$ and $R_{a v}(i)$ is the average route length for generation $i$. Note that this function emphasizes more the notion of population fitness than the best individual fitness. In terms of pure combinatoric optimization knowing that there exists a unique optimum state it is of course finding the fittest individual in the shortest amount of time that is of interest. However, in many other problems, for instance in evolution and in other problems with a time dependent environment what is important is fitness of the population. The above fitness function takes into account both convergence speed and average population fitness.

\section{Results}

In Figure 5 we see the results of various GAs in the landscape of Figure 2. The plot shows in the upper half relative frequency of the optimum string as a function of time and in the lower half the average values of the codified GA parameters as a function of time. The initial population was chosen at random. Note that the population size here is large compared to the size of the state space as our intention was to investigate the effects of parameter codification without strong finite size effects complicating the picture due to sampling errors. We will consider the latter thoroughly in a future publication.

The most notable feature of Figure 5 is the behaviour of the coded parameters. For mutations we see that the system begins to "cool" itself down as the population begins

to order. It is clear that there is no direct selective benefit in a given generation for one 
mutation rate versus another, however, we do see quite clearly the effects of symmetry breaking in that strings with low mutation rates are likely to have fitter offspring. This symmetry breaking becomes more pronounced as a function of time. With respect to crossover we see that there is rather a tendency to increase than decrease as a function of time. One can understand this from the point of view that crossover is strictly neutral for the fully ordered component of the population, i.e. all the optimum strings, whilst a high crossover rate helps in getting rid of unfit strings as found in [10]. In terms of comparison with a fixed parameter GA we see that an 8-bit encoded GA spends more time trying to find the optimum mutation and crossover rates as it has to search through more possibilities. Note that the steady state population for a codified mutation rate will always be superior to that of a fixed rate simply because the population can only be strictly ordered when $p=0$.

In Figure 6 we show what happens with the landscape in Figure 2 but now in the case where the initial population is totally concentrated at one point, 49. One might ask why we would want to consider such a case. The reason why will become more obvious after seeing the results from the jumper landscape. Suffice it to say here that in a time dependent landscape it might well be that the population has pretty much converged to an optimum but that at a certain point the landscape changes such that the original global optimum is now only a local optimum and the system must then seek the new global optimum. If the landscape changes only after the population has converged to the original optimum then in terms of evolution in the new landscape the system is starting from a very special initial condition.

Considering the explicit results we see that a fixed parameter GA performs particularly badly. The reason why is simple: crossover does not act very efficiently in encouraging diversification when one starts with an ordered population. This role has to be played by mutation. If the mutation rate is low then the search time to find the optimum is large. It is clear that codifying crossover and not mutation does not help. When mutation is codified the results are clearly far superior. Once again we see how the system cools itself down after the GA begins to find the optimum. The behaviour of the crossover probability as a function of time is interesting in that it shows an increase even though we have emphasized that crossover is not very efficient. The point is, that despite its inefficiency it is still a positive operator in that it aids the search for the new optimum. Only in the case of zero mutation rate and a totally ordered population is it strictly neutral.

The next figure, Figure 7, shows the results associated with the deceptive landscape of Figure 3. The initial population was biased with 100 individuals being placed at each optimum to make the problem even more "deceptive". One sees that the fixed parameter GA was incapable of maintaining the population at the global optimum. The 8-bit coded GA by contrast increased the relative concentration of the optimum without any problems. The 3-bit coded GA sometimes converged to the global optimum and sometimes to the local one. Once again we see that in terms of mutations the system cools itself down. The most interesting result here however is what happens to the crossover rate. In the results from the non-deceptive landscape the crossover probability 
rose monotonically. Here, however, we see that initially there is a very sharp drop in the rate. This is a direct response to the deception. Crossover of 0 strings with 63 strings produces very unfit results. As the system starts with a large number of them those that are coded with low crossover rates will be preferred. Eventually as the system begins to order around the global optimum crossover loses its destructive nature and so the net rate increases.

Figure 8 contains the results for the deceptive landscape when $p=0$. In this case we started with an initial population of 80 individuals, 20 of which were located at one optimum, 20 at the other and the remaining 40 distributed randomly between them. Once again, initially, due to the large populations associated with the two optima, crossover is very destructive as any crossover which includes strings from both optima will result in very unfit offspring. Note that the average population fitness first decreases somewhat although this decrease is minimal compared to the fitness decrease in the fixed parameter GA.

In Figure 9 we see what happens for the jumper landscape. The upper curves show the relative frequencies of the optima using 8-bit and 3-bit codification and also what happens when $p_{c}=0$ and only the mutation rate is coded. There are several notable features: first of all we see that the fixed parameter GA was incapable of finding the new optimum whereas the coded GAs had no problem whatsoever. For the case $p_{c}=0$ the curve 40,41 shows the relative frequency of the strings associated with the optimum at 40 and 41. Before the landscape "jump" this optimum is local being less fit than the global optimum at 10 and 11. After the "jump" it is fitter but less fit than the new global optimum 63 which is an isolated point. One thus sees that the optimum was found in a two-step process after the landscape change. First the strings started finding the optima 40, 41 before moving onto the true global optimum, 63. Immediately after the jump the effective population of the new global optimum is essentially zero. The number of strings associated with 40 and 41 first starts to grow substantially at the expense of 10 and 11 strings. At its maximum the number of optimum strings is still very low, however, very soon thereafter the algorithm manages to find the optimum string which then increases very rapidly at the expense of the rest. The striking result here can be seen by comparing the changes in the relative frequencies with the changes in the average mutation rate, especially in the case $p_{c}=0$. Clearly they are highly correlated. First, while the population is ordering itself around the original optimum, there is an effective selection against high mutation rates as one can see by the steady decay of the average mutation rate. After the jump one can see a noticeable increase in the mutation probability as the system now has to try to find fitter strings. As the global optimum is an isolated state it is much easier to find fit strings associated with 41 and 40 . The system now thinks that this is the optimum and starts to cool down again only to find that this was not so, whereupon the system heats up again to aid the removal of the population to the true global optimum. It is clear that there is a small delay between the population changes and changes in the mutation rate. This is only to be expected given that there is no direct selective advantage in a given generation for a particular mutation rate. The selective advantage of a more mutable genotype over a less mutable 
one can only come about via a feedback mechanism. It is precisly this feedback process that is described and measured by the effective fitness function. The average mutation rate also grows due to another effect which is that the new optimum is more likely to be reached by strings with high mutation rates which then grow strongly due to their selective advantage. Thus high $p$ strings will naturally dominate the early evolution of the global optimum. After finding the optimum however it will become disadvantageous to have a high mutation rate hence low mutation strings will begin to dominate.

We now turn to the case of the TSP. In Figure 10 we see a comparison between GA performance for fixed rates, $p=0.1, p_{c}=0.2$, and coded rates. One can see that in terms of convergence velocity the two are roughly comparable, however in terms of the algorithm fitness function, (22), the coded algorithm performs much better due to the fact that the average member of the population is almost as good as the best. The relative performances are shown in Figure 11. The shortest route for this particular problem reported in [16 is 10930. The best result from our GA is 11,040 for fixed parameters and 11,662 for coded parameters. We found that inversion affects the GA's performance more than mutation due to its more global scope.

In Figure 12 we see the results for the jumper TSP landscape and in Figure 13 the efficiencies of the two GAs are compared. Finally in Figure 14 we see the evolution of the coded crossover rate in the non-jumper and jumper landscapes. Note that very soon after the landscape change at $t=3000$ the crossover rate begins to rise reaching a peak at $t=3500$ where it is almost double its prejump value. Thus as in the case of the previous jumper landscape one sees that the parameters respond to changes in the landscape. In this case it is the inversion rate which rises after the landscape change in order to aid the algorithm in the search for the new optimum.

\section{Discussion and Conclusions}

In this paper we have investigated the effects of coding the probabilities that govern the action of genetic operators in a simple GA based on a select set of model fitness landscapes. One of the basic premises of the paper was to investigate how parameter codification may help in adaptive search problems and to illuminate some of the consequent theoretical issues.

Optimizing the exogeneous parameters of a GA is a very tricky business that at the end of the day must be landscape dependent. Generically this will also mean time dependent. We have therefore tried to show what one might gain via a codification of these parameters within the chromosomes themselves thus leading to an essentially, totally autonomous system. We saw that in a time-dependent landscape certain parameter values will be favoured over others in order to facilitate the search for new optima, and that the values themselves will change over time. However, the chief lessons we wish to point out from our work here have much wider implications than those to be drawn from a simple comparison of coded versus non-coded GA performance.

What are these lessons? There are two: the lesson of induced symmetry breaking,

and the lesson of effective fitness. In the former, if we have a degenerate genotype- 
phenotype mapping, i.e. there exist synonymous genotypes, then there exists a symmetry, or perhaps better to say an equivalence relation on $G$. By degenerate we mean explicitly that the different genotypes all correspond to exactly the same phenotypic fitness value. The fitness landscape as a function of genotype thus has neutral directions. In the presence of pure selection the system will be unable to distinguish between these directions. However, on including mutation and/or crossover this degeneracy will be lifted, certain directions now being preferred over others even though there is no direct selective advantage for them. Thus we may say there is an induced symmetry breaking. The toy model of section 3 and the later numerical results all confirm this clearly

and explicitly. In understanding this phenomenon the normal concept of fitness is of little use, which brings us to the second important lesson, that of effective fitness. Even though certain directions in $G$ may be neutral in terms of fitness they most definitely are not in terms of the effective fitness. We therefore claim that effective fitness, and the consequent fitness landscape in terms of it, are much more relevant and meaningful concepts in the presence of other genetic operators such as mutation and crossover. The effective landscape will be time dependent even though the original landscape wasn't. We will give a more thorough analysis of the effective fitness in a future publication.

Also of interest is the relation to the theory of natural evolving systems. There the bare mutation rates have a biophysical origin which cannot be coded directly, however it is also true that different synonymous codons can have varying mutabilities. The most obvious reason for this is that point mutation itself does not respect the equivalence between synonyms. For example, in the case of the six leucine codons, CTPu have four possible silent point mutations, CTPy have three and TTPu have two. Therefore, if missense mutations are selected negatively then one will find that $C T P u$ codons have an effective selective advantage due to their higher resistance to mutation. Vice-versa, in the recognition regions of the enveloping protein of a lentivirus there is a preference for codons that mutate non-synonymously, in order to escape detection by the immune system [17]. One can view this effect as an example of self-adaptation where the choice of mutation rate is realized indirectly through the codon bias.

\section{Acknowledgments}

This work was partially support by a CONACYT scholarship to J. Mora-Vargas and by DGAPA grant IN105197.

\section{References}

[1] S. A. Kauffman, The Origins of Order, Oxford University Press, Oxford (1993).

[2] T. Bäck, Evolutionary Algorithms in Theory and Practice: evolution strategies, evolutionary programming, genetic algorithms, (Oxford Univ. Press 1996).

[3] D.B. Fogel, System Identification through Simulated Evolution: A Machine Learning Approach to Modeling, (Ginn Press, Needham, MA, 1991). 
[4] Handbook of Genetic Algorithms L. Davis, (ed.) (Van Nostrand Reinhold, New York 1991); Holland, J.H., Scientific American, July 1992, 66-72.

[5] K. De Jong, An Analysis of the Behaviour of a Class of Genetic Adaptive Systems, Ph.D thesis, (Univ. of Michigan, 1975); J.D. Schaffer, R.A. Carauna, L.J. Eshelman and R. Das, Proceedings of the Third Int. Conf. on Genetic Algorithms and their Applications, 51-60 (Morgan Kaufman 1989); J.J. Grefenstette, IEEE Transactions on Systems, Man and Cybernetics, SMC-16(1), 122 (1986).

[6] W.E. Hart and R.K. Belew, Proceedings of the Fourth Int. Conf. on Genetic Algorithms and their Applications, 190-195 (Morgan Kaufman 1991).

[7] H.P. Schwefel, Numerical Optimization of Computer Models, (J.P. Wiley 1981).

[8] T. Bäck, Parallel Problem Solving from Nature 2, ed.s R. Männer and B. Manderick, (Elsevier 1992) 85; Proceeding of the First European Conference on Artificial Life, ed.s F.J. Varela and P. Bourgine, (MIT Press 1992) 263.

[9] J.D. Schaffer and A. Morishima, Proceedings of the Second Int. Conf. on Genetic Algorithms and their Applications, 36-40 (Lawrence Erlbaum Associates 1987)

[10] C.R. Stephens and H. Waelbroeck, Analysis of the Effective Degrees of Freedom in Genetic Algorithms, ICN, UNAM preprint: ICN-UNAM-96-08; adap-org/9611005; Proceedings of the Sixth Int. Conf. on Genetic Algorithms and their Applications, (Morgan Kaufman 1997).

[11] D. E. Goldberg, Genetic Algorithms in search, optimization and machine learning, Addison Wesley, Reading, MA (1989).

[12] A.H. Wright, Foundations of Genetic Algorithms, 205 (Morgan Kaufmann 1991).

[13] R.A. Caruna and J.D. Schaffer, Proceedings of the 5th Int. Conf. on Machine Learning, 153 (Morgan Kaufmann 1988).

[14] M. Kimura, The Neutral Theory of Molecular Evolution, Cambridge University Press, Cambridge (1983).

[15] A. Homaifar, S. Guan and G.E. Liepins, Proceedings of the?? Int. Conf. on Genetic Algorithms and their Applications, (Morgan Kaufman 198?)

[16] R. L. Karg and G. L. Thompson, Management Science 10, 225 (1964).

[17] H. Waelbroeck, Codon Bias and Mutability in HIV Sequences, National University of Mexico Preprint ICN-UNAM-97-09 (adap-org/9707) (1997). 


\section{Figure Captions}

Figure 1: Graph of relative frequency as a function of time. The squares represent genotype $\mathrm{C}$, the diamonds genotype $\mathrm{A}$ and the crosses genotype $\mathrm{B}$.

Figure 2: Graph of fitness versus genotype.

Figure 3: Graph of fitness versus genotype for "deceptive" landscape.

Figure 4: Graph of fitness versus genotype after "jump" from Figure 2.

Figure 5: Graph of relative concentration of the optimum (CR) and average crossover and mutation probabilities as a function of time. CR is shown in the upper half and the average rates in the lower half. CR-FR is the curve for a fixed rate GA; CR-3b for coded 3-bit probabilities; CR-8b for coded 3-bit probabilities; Mut 3b, Mut 8b, Cross $3 \mathrm{~b}$ and Cross $8 \mathrm{~b}$ are the average mutation and crossover probabilities in 3-bit and 8-bit representations.

Figure 6: The same as in Figure 5, except now the initial population is centered at 49 in the landscape of Figure 2. CR-Cro 3b represents a GA where $p$ is fixed at 0.01 and crossover is 3 -bit coded. Cros $3 \mathrm{~b}$ is the corresponding average crossover rate.

Figure 7: Same curves as in Figure 5 but now for the "deceptive" landscape of Figure 3 and with different initial population.

Figure 8: Graph of relative concentration and average crossover probabilities as a function of time for the landscape of Figure 3 with initial population size of 80 and $p=0$. CR-Fit.1 is the relative concentration of the global optimum at point 0; CR-Fit.2 is the relative concentration of the local optimum at point 63; Avg. Cross is the average crossover rate and Avg. Fit the average population fitness.

Figure 9: Graph of relative concentration of the optimum (CR) and average crossover and mutation probabilities as a function of time for the "jumper" landscape. CR-3b and CR-8b are the results for 3-bit and 8-bit encoded algorithms CR-Mut8b is the result for coded mutation with $p_{c}=0$, with 40,41 being the relative concentration of strings associated with the local optimum at 40 and 41. Mut 3b, Mut 8b, Cross 3b and Cross 8b are the average mutation and crossover probabilities in 3-bit and 8-bit representations. The solid line for Mut8b is the average mutation rate in the case $p_{c}=0$.

Figure 10: Graph of Fitness (Route length) versus time for 33-city TSP. f2.fit and f2.bes represent the average and best fitnesses for a fixed parameter GA whilst s2.fit and s2.bes are for a coded 8-bit GA.

Figure 11: Graph of Efficiency versus time for fixed parameter and coded parameter GAs in the TSP; f2.eff for the fixed and s2.eff for the coded GA.

Figure 12: Graph of Fitness (Route length) versus time for "jumper" TSP. f2.fit and 
f2. bes represent the average and best fitnesses for a fixed parameter GA whilst s2.fit and s2.bes are for a coded 8-bit GA.

Figure 13: Graph of Efficiency versus time for fixed parameter and coded parameter GAs in the "jumper" TSP; fj2.eff for the fixed and sj2.eff for the coded GA.

Figure 14: Graph of Crossover rate versus time for the 33-city TSP and the "jumper" TSP. sj2.cro is for the "jumper" landscape 